\title{
The Impact of Individual Health Education on Health Literacy: Evalu- ation of the Translated Version (Sinhala) of Health Education Impact Questionnaire in Type 2 Diabetes
}

\section{Bulathsinghalage Poornima Reshamie Cooray ${ }^{1}$, Hana Morrissey ${ }^{2 *}$, Eisha Indumani Waidyarathne ${ }^{3}$, Patrick Anthony Ball ${ }^{2}$ and Manilka Sumanathilake ${ }^{4}$}

${ }^{1}$ Department of Pharmacy, University of Ruhuna, Sri Lanka

${ }^{2}$ School of Pharmacy, University of Wolverhampton, United Kingdom

${ }^{3}$ Medical Faculty, University of Ruhuna, Sri Lanka

${ }^{4}$ Diabetes and Endocrinology Unit, Teaching Hospital Karapitiya, Sri Lanka

*Corresponding author: Dr. Hana Morrissey, Faculty of Science and Engineering, School of Pharmacy, University of Wolverhampton, Wulfruna Street, Wolverhampton, WV1 1LY, United Kingdom, Tel: +44-(0)-1902-322173, E-mail: Hana.

Morrissey@wlv.ac.uk

\begin{abstract}
Introduction: Type 2 diabetes mellitus and its complications is increasingly prevalent in Sri Lanka. Patients with low health literacy worsen the glycaemic control and diabetes complications. However, lack of studies on the effect of health literacy that affects clinical health outcomes and the effect of health education on it.
\end{abstract}

Aim: This study aimed to evaluate the effect of health education impact questionnaire, translated into Sinhala is an appropriate tool to evaluate the impact of health education program for type 2 diabetes patients based on literacy improvement.

Methods: This project was designed as a preliminary case-control study, with health education as the main intervention. Repeated health-education interventions were compared to the control group at baseline, followed up at six and 12 months. The improvements in knowledge assessed through health education impact questionnaire that was translated and culturally adapted to Sinhala language.

Results: The study outcomes from 150 patients reveal that all heiQ ${ }^{\mathrm{TM}}$ domains showed at least low to moderate correlations with the follow ups in intervention while, small effect had been shown in health service navigation in control group.

Conclusion: This study reveals that the translated version of health education questionnaire well adapted in assessing knowledge improvement among Sri Lankan participants with type 2 diabetes.

\section{Keywords}

Type 2 diabetes mellitus, Health education impact questionnaire, HeiQ, Health education

\section{Introduction}

Type 2 diabetes (T2DM) is a common chronic condition which, if not controlled, can cause complications such as nephropathy, retinopathy and neuropathy. In recent years T2DM prevalence increased globally. There were 1.16 million cases of all types of diabetes were recorded in Sri Lanka in 2016 as stated in an International Federation of Diabetes Atlas [1-3]. A significant reduction in diabetes-related complications has been recorded with the diabetes education in primary care setting, with the consideration of health beliefs and their cultural behaviours [2-4].

However, a range of indices are used to measure health education in individuals and populations. Due to use of different, new or non-validated tools by researchers to assess the health education impact; there were few available evidence of a direct relationship between health education and improvement of patients' health outcomes [5-7].

Citation: Cooray BPR, Morrissey H, Waidyarathne El, Ball PA, Sumanathilake M (2018) The Impact of Individual Health Education on Health Literacy: Evaluation of the Translated Version (Sinhala) of Health Education Impact Questionnaire in Type 2 Diabetes. Int J Diabetes Clin Res 5:087. doi.org/10.23937/23773634/1410087

Received: February 26, 2018: Accepted: May 12, 2018: Published: May 14, 2018

Copyright: (C) 2018 Cooray BPR, et al. This is an open-access article distributed under the terms of the Creative Commons Attribution License, which permits unrestricted use, distribution, and reproduction in any medium, provided the original author and source are credited. 
The health education impact questionnaire (heiQ ${ }^{\mathrm{TM}}$ ) is a validated tool that has been in use to evaluate the impact of patient education interventions on patient knowledge, behaviours, ability to act in an emergency, to navigate the health services and resourcefulness $[8,9]$. The questionnaire has been used to measure health education impact on patients with a broad range of chronic diseases and validated in different settings, by a range of health professionals, which has been adapted to 20 languages [9-11].

The heiQ ${ }^{\mathrm{TM}}$ was derived from a grounded approach, with its most prominent feature being its breadth and capability to evaluate individual's ability to manage their condition irrespective of which chronic disease they have. This questionnaire covers eight main domains in 40 questions: Health directed behaviour, positive and active engagement in life, emotional wellbeing, self-monitoring and insight, constructive attitudes and approaches, skills and technique acquisition, social integration and support and health services navigation and additionally with another section to evaluate the progress of the education program [10-12].

Jayasuriya, et al. 2015 study concluded that improvement in glycaemic control was significant among Sri Lankan patients if they were modified through diabetes self-management interventions as in many other countries [4]. However, Sri Lankans patients have poor understanding of the importance of dietary control, physical activity and adherence to therapy in the management of diabetes. This might be due to insufficient information they receive from their treating professionals [2,13-15]. Additionally, Sri Lanka has remote and rural locations where government-specialist clinics are scarce. Those government specialist medical clinics available in urban areas are often overcrowded with patients. Thus, most T2DM patients are managed in primary care system, by general practitioners [13-15].

However, Jayasuriya, et al., 2015 and a systemic review of Cooray, et al., 2017 revealed that there is a gap between the patient knowledge, management of diabetes and the effectiveness of patient education measured by validated questionnaires in Sri Lanka $[4,16,17]$.

Therefore, the aim of this study was to evaluate the impact of structured health education program on patients' knowledge by utilizing the heiQ questionnaire on their health-related behaviours, capabilities and motivations.

\section{Design and Methods}

This study was designed as a prospective, non-experimental, step-up controlled study, with health education as the main intervention (Figure 1). To reduce samples cross contamination between the non-intervention and intervention groups cluster design method has been adopted. Thus, sample was recruited from two main independent tertiary care facilities in Western and Southern Provinces of Sri Lanka.

Both non-intervention and intervention groups participants continued to receive their usual diabetes care and treatment as indicated by their treating practitioners and the local guidelines for each of the two hospitals.

Potential participants were informed verbally and in writing about the study objectives and processes, their rights, and what was expected of them if they decided to participate. Participation was voluntary, with written consent obtained in Sinhala, their native language prior to participation.

The heiQ ${ }^{\mathrm{TM}}$ instrument was translated (forwardbackward translation) to Sinhala by professional translation services contracted by the developer, Osborne, et al. at the University of Melbourne. The heiQ ${ }^{\mathrm{TM}}$ was used to measure participants' knowledge and attitude towards their self-care, pre- and post- the delivery of a structured health-education session and compared with non-intervention group. The health education session was carried out one-on-one, verbal script-based consultation, aided by PowerPoint presentation by the

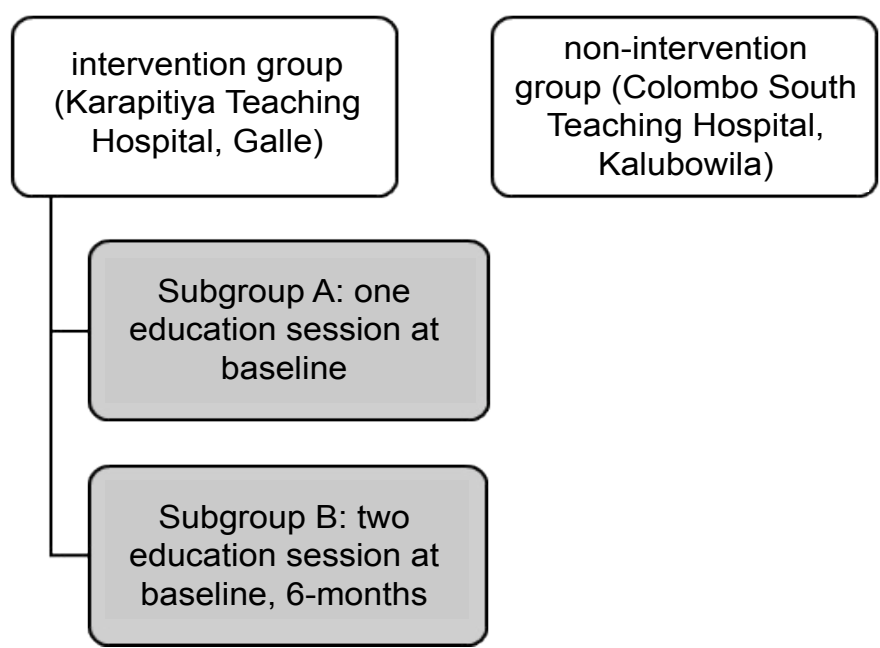

Figure 1: Sample design. 
primary investigator. The education program followed the Pathophysiology, Indication, Treatment and Specifics (PITS) patient education model [17]. The model presents information in an organised and logical format that enhances the receiver's ability to recall information. Also, this model enables the receiver to easily follow the educators' thoughts and direction of information flow, which may be contingent on the emotional state of the patient or other constraints [17].

\section{Sample selection, control and randomisation}

All genders with T2DM patients aged 18 years and over were approached regardless of level of education, socioeconomic level and employment status. As people under the age of 18 and those who are pregnant or diagnosed with polycystic ovarian syndrome or gestational diabetes or have severe diabetes complications who require different type of educational material and higher level of consent, they were excluded from the study.

Participant was enrolled if they were diagnosed with T2DM by the physicians who was not on haemodialysis and able to speak and understand Sinhala, who is not identified as having cognitive, hearing or vision impairment.

The study participants were approached on the basis of every third T2DM patient from the clinic registry for both non-intervention and intervention groups in two different tertiary care facilities in Sri Lanka, at the beginning of the study. Then, intervention group further categorised into subgroups (Subgroup A and Subgroup B) and it was carried out using a computer-generated randomising algorithm in Microsoft Excel ${ }^{\mathrm{TM}}$.

Being a low-budget preliminary study, a total of 166 participants was considered as a manageable sample that would provide sufficient data to inform future studies on the trends, variances and relationships, between self-care behaviour, level of health education and diabetes control. The estimated withdrawal rate for the study was expected to be approximately $10 \%$.

\section{Data collection and analysis}

Demographic data were collected using a socio-demographic survey questionnaire designed by the investigator. The research method was based on two main aspects the clinical data collection and the diabetes health-education intervention. Clinical data collection took place on three occasions for all intervention and non-intervention participants - at the enrolment day, six-months and 12-months follow up - while diabetes education was carried out on the following basis:

- At the enrolment day for intervention group (Subgroups A and B)

- At the six-month follow up for participants in Subgroup B only.

Clinical and biomedical data collection was confined to measuring weight, height, waist circumference, $\mathrm{BP}$, and blood sample collection for HbA1c and lipid profile tests.

Change in medications was not in the scope of this study; however patient adherence to therapy and selfcare behaviours were monitored during the two follow up visits at six and 12 months.

The heiQ ${ }^{\mathrm{TM}}$ validated instrument used under the licensed agreement with Deakin University, Australia and printed heiQ ${ }^{\mathrm{TM}}$ baseline questionnaires were administered at the data collection sites; supported (explained without input) by the investigator, to measure the knowledge about their disease condition, and medication adherence before the intervention. Patients were asked to tick the most applicable answer describing their level of knowledge. The participants viewed the questions without the domain subtitle, allowing them to answer the individual questions based on their own perspective without being influenced by the domain title.

Following the completion of the questionnaire, the intervention group received the structured health-education program on one-on-one basis by the principal investigator. The program comprised of a presentation covering the pathogenesis, progression and complications of T2DM; importance of self-management and physicians follow-up. The session then progressed to focus on the participant prescribed medication, including the use of blood glucose monitors and insulin pens, how the medication work, their side effects and the benefit from adherence to medication on disease prognosis and development of complications. The same procedure was used for the data collection process of the non-intervention group with the exclusion of the diabetic health-education intervention. All enrolled participants (Subgroup A and B of intervention group and the non-intervention group) completed the heiQ ${ }^{\text {TM }}$ follow up at 6 months and at twelve months, which included patient feedback on the education program and the way it was delivered. Completed questionnaires with their ID numbers were analysed and scored based on a Likert-type scale from 1 to 4 , corresponding to <strongly disagree>, <disagree>, <agree> and <strongly agree>. Each domain has four to six questions. The scores were summated within each domain to obtain a scale score for each domain based on guidelines adopted by the questionnaire developer.

The participants' domain mean scores achieved in the baseline were compared to the follow-up mean scores and with non-intervention group to determine if their knowledge improved after the delivery of the patient education intervention using developers' guideline on effect size calculation and Analysis of Covariance (ANCOVA) using IBM ${ }^{\circledR}$ SPSS $^{\circledR}$ (version 23) and R-Studio ${ }^{\circledR}$ (version 3.2.2) software packages.

\section{Results}

Overall analysis of demographics of 150 patients who 
were retained (out of 166 participants) at the end of study (retained rate $92 \%$ ) indicate that large percentage of participants were women $(72.3 \%, 120)$ with mean age of 56.2 years, with a standard deviation [SD] of 8.95. Most participants (85\%) were managing their diabetes with oral hypoglycaemic agents. Among these patients, three-quarters of the T2DM patients were treated with lipid lowering agents, while half of the T2DM patients also received treatment for hypertension. In addition to dyslipidaemia and hypertension, a few participants were treated for other conditions such as thyroid disorders (three patients), arthritis (three patients) and one patient had cancer.

Table 1 summarises the comparison of categorical and important biomarker baseline values for intervention and non-intervention groups. Detailed baseline values for each subgroup levels publish in a separate manuscript.

The analysis of heiQ ${ }^{\mathrm{TM}}$ based on the developers' guidelines indicate that one hundred and sixty-four heiQ $^{\mathrm{TM}}$ forms were completed at baseline, 152 (92.7\%) at the six months' follow-up and $150(91.5 \%)$ at the end of 12 months' follow-up.

The results of this study based on analysis of covariance (ANCOVA) indicated significant improvement of the knowledge in all other domains between baseline to six months, six to twelve months and baseline to twelve

Table 1: One-Way ANOVA results for comparing the mean measurement values of intervention group vs. non-intervention group at baseline analysis.

\begin{tabular}{|c|c|c|c|c|c|c|}
\hline \multirow[t]{2}{*}{ Measurement } & \multicolumn{2}{|c|}{ Intervention } & \multicolumn{2}{|c|}{ Non-intervention } & \multirow[t]{2}{*}{ F value } & \multirow[t]{2}{*}{ Sig. } \\
\hline & Mean & $\begin{array}{l}\text { Standard } \\
\text { Deviation (SD) }\end{array}$ & Mean & $\begin{array}{l}\text { Standard } \\
\text { Deviation (SD) }\end{array}$ & & \\
\hline Age (yr) & 56 & 8.9 & 56 & 9.2 & 0.669 & 0.514 \\
\hline Weight (kg) & 59.0 & 10.3 & 61.0 & 9.1 & 0.954 & 0.387 \\
\hline Height (cm) & 154.4 & 8.4 & 157.3 & 7.6 & 3.185 & $0.044^{*}$ \\
\hline Waist Circumference $(\mathrm{cm})$ & 95.5 & 9.1 & 96.8 & 7.9 & 0.598 & 0.551 \\
\hline HbA1c level (\%) & 8.56 & 1.62 & 8.65 & 1.53 & 0.279 & 0.757 \\
\hline Systolic Pressure (mmHg) & 126.3 & 16.8 & 129.4 & 16.7 & 0.651 & 0.523 \\
\hline Diastolic Pressure $(\mathrm{mmHg})$ & 79.6 & 9.4 & 84.9 & 9.1 & 5.970 & $0.003^{* *}$ \\
\hline Heart rate (bpm) & 78.4 & 11.6 & 81.1 & 11.0 & 1.899 & 0.153 \\
\hline Total Cholesterol (mg/dL) & 189.8 & 44.1 & 181.3 & 38.9 & 0.908 & 0.406 \\
\hline HDL Cholesterol (mg/dL) & 45.1 & 8.5 & 45.0 & 8.9 & 0.097 & 0.908 \\
\hline Triglycerides (mg/dL) & 126.3 & 64.4 & 129.1 & 46.7 & 0.250 & 0.779 \\
\hline LDL Cholesterol (mg/dL) & 100.1 & 35.2 & 109.5 & 35.9 & 1.330 & 0.267 \\
\hline BMI Value $\left(\mathrm{kg} / \mathrm{m}^{2}\right)$ & 24.8 & 4.3 & 24.7 & 3.5 & 0.075 & 0.928 \\
\hline
\end{tabular}

*Between group effect is statistically significant at the $p<0.05$ level; "*Between group effect is statistically significant at the $p<$ 0.01 level.

Table 2: Summary of between-intervention and non-intervention group effects for eight domains at baseline, 6 months and 12 months.

\begin{tabular}{|c|c|c|c|c|c|c|}
\hline Domains & Time interval (months) & Groups & Mean value & F Statistic & Significance & Size of Effect \\
\hline \multirow{6}{*}{$\begin{array}{l}\text { Health directed } \\
\text { behaviour }\end{array}$} & \multirow[t]{2}{*}{$0-6$ months } & Intervention & 2.82 & \multirow[t]{2}{*}{24.25} & \multirow[t]{2}{*}{$<0.001^{* *}$} & \multirow[t]{2}{*}{0.138} \\
\hline & & Non-intervention & 2.73 & & & \\
\hline & \multirow[t]{2}{*}{ 6-12 months } & Intervention & 3.09 & \multirow[t]{2}{*}{65.75} & \multirow[t]{2}{*}{$<0.001^{* *}$} & \multirow[t]{2}{*}{0.309} \\
\hline & & Non-intervention & 2.65 & & & \\
\hline & \multirow[t]{2}{*}{$0-12$ months } & Intervention & 3.35 & \multirow[t]{2}{*}{80.78} & \multirow[t]{2}{*}{$<0.001^{\star \star}$} & \multirow[t]{2}{*}{0.355} \\
\hline & & Non-intervention & 2.68 & & & \\
\hline \multirow{6}{*}{$\begin{array}{l}\text { Positive } \\
\text { and active } \\
\text { engagement in } \\
\text { life }\end{array}$} & \multirow[t]{2}{*}{$0-6$ months } & Intervention & 2.84 & \multirow[t]{2}{*}{15.88} & \multirow[t]{2}{*}{$<0.001^{* *}$} & \multirow[t]{2}{*}{0.095} \\
\hline & & Non-intervention & 2.80 & & & \\
\hline & \multirow[t]{2}{*}{ 6-12 months } & Intervention & 3.09 & \multirow[t]{2}{*}{13.89} & \multirow[t]{2}{*}{$<0.001^{* *}$} & \multirow[t]{2}{*}{0.086} \\
\hline & & Non-intervention & 2.83 & & & \\
\hline & \multirow[t]{2}{*}{$0-12$ months } & Intervention & 3.20 & \multirow[t]{2}{*}{23.07} & \multirow[t]{2}{*}{$<0.001^{* *}$} & \multirow[t]{2}{*}{0.135} \\
\hline & & Non-intervention & 2.86 & & & \\
\hline \multirow{6}{*}{$\begin{array}{l}\text { Self-monitoring } \\
\text { and insight }\end{array}$} & \multirow[t]{2}{*}{$0-6$ months } & Intervention & 2.97 & \multirow[t]{2}{*}{13.27} & \multirow[t]{2}{*}{$<0.001^{* *}$} & \multirow[t]{2}{*}{0.081} \\
\hline & & Non-intervention & 2.96 & & & \\
\hline & \multirow[t]{2}{*}{ 6-12 months } & Intervention & 3.14 & \multirow[t]{2}{*}{23.09} & \multirow[t]{2}{*}{$<0.001^{* *}$} & \multirow[t]{2}{*}{0.137} \\
\hline & & Non-intervention & 2.89 & & & \\
\hline & \multirow[t]{2}{*}{$0-12$ months } & Intervention & 3.21 & 28.16 & $<0.001^{* *}$ & 0.161 \\
\hline & & Non-intervention & 2.82 & & & \\
\hline
\end{tabular}




\begin{tabular}{|c|c|c|c|c|c|c|}
\hline \multirow[t]{5}{*}{$\begin{array}{l}\text { Constructive } \\
\text { attitudes \& } \\
\text { approaches }\end{array}$} & $0-6$ months & $\begin{array}{l}\text { Intervention } \\
\text { Non-intervention }\end{array}$ & $\begin{array}{l}2.92 \\
2.96\end{array}$ & 45.11 & $<0.001^{* *}$ & 0.229 \\
\hline & \multirow[t]{2}{*}{ 6-12 months } & Intervention & 3.16 & \multirow[t]{2}{*}{7.83} & \multirow[t]{2}{*}{$0.006^{* *}$} & \multirow[t]{2}{*}{0.050} \\
\hline & & Non-intervention & 2.81 & & & \\
\hline & \multirow[t]{2}{*}{$0-12$ months } & Intervention & 3.23 & \multirow[t]{2}{*}{14.83} & \multirow[t]{2}{*}{$<0.001^{* *}$} & \multirow[t]{2}{*}{0.091} \\
\hline & & Non-intervention & 2.84 & & & \\
\hline \multirow{6}{*}{$\begin{array}{l}\text { Skills and } \\
\text { technique } \\
\text { acquisition }\end{array}$} & \multirow[t]{2}{*}{$0-6$ months } & Intervention & 2.78 & \multirow[t]{2}{*}{ n.s. } & \multirow[t]{2}{*}{ n.s. } & \multirow[t]{2}{*}{ n.s. } \\
\hline & & Non-intervention & 2.75 & & & \\
\hline & \multirow[t]{2}{*}{ 6-12 months } & Intervention & 3.10 & \multirow[t]{2}{*}{29.14} & \multirow[t]{2}{*}{$<0.001^{* *}$} & \multirow[t]{2}{*}{0.165} \\
\hline & & Non-intervention & 2.95 & & & \\
\hline & \multirow[t]{2}{*}{ 0-12 months } & Intervention & 3.23 & \multirow[t]{2}{*}{32.98} & \multirow[t]{2}{*}{$<0.001^{* *}$} & \multirow[t]{2}{*}{0.182} \\
\hline & & Non-intervention & 2.68 & & & \\
\hline \multirow{6}{*}{$\begin{array}{l}\text { Social integration } \\
\text { \& support }\end{array}$} & \multirow{2}{*}{ 0-6 months } & Intervention & 2.86 & \multirow[t]{2}{*}{13.21} & \multirow[t]{2}{*}{$<0.001^{* *}$} & \multirow[t]{2}{*}{0.080} \\
\hline & & Non-intervention & 2.84 & & & \\
\hline & 6-12 months & Intervention & 3.11 & 14.55 & $<0.001^{* *}$ & 0.089 \\
\hline & & Non-intervention & 2.86 & & & \\
\hline & $0-12$ months & Intervention & 3.17 & 21.39 & $<0.001^{* *}$ & 0.126 \\
\hline & & Non-intervention & 2.90 & & & \\
\hline Health services & $0-6$ months & Intervention & 2.66 & 11.28 & $0.001^{* *}$ & 0.092 \\
\hline navigatıon & & Non-intervention & 2.64 & & & \\
\hline & 6-12 months & Intervention & 3.15 & 24.22 & $<0.001^{* *}$ & 0.141 \\
\hline & & Non-intervention & 2.85 & & & \\
\hline & 0-12 months & Intervention & 3.14 & 31.39 & $<0.001^{* *}$ & 0.176 \\
\hline & & Non-intervention & 2.74 & & & \\
\hline Emotional & $0-6$ months & Intervention & 2.40 & 15.28 & $0.001^{* *}$ & 0.092 \\
\hline & & Non-intervention & 2.41 & & & \\
\hline & 6-12 months & Intervention & 2.13 & 17.11 & $<0.001^{\star *}$ & 0.107 \\
\hline & & Non-intervention & 2.45 & & & \\
\hline & 0-12 months & Intervention & 2.01 & 21.91 & $<0.001^{* *}$ & 0.133 \\
\hline & & Non-intervention & 2.44 & & & \\
\hline
\end{tabular}

*The mean difference is significant at the 0.05 level; n.s. not significant.

Table 3: Summary of between-intervention subgroups ( $A$ and $B$ ) and non-intervention group effects for eight domains at baseline to 12 months.

\begin{tabular}{|c|c|c|c|c|c|c|c|c|}
\hline \multirow[b]{3}{*}{ Domain } & \multicolumn{5}{|c|}{ Intervention group } & \multirow{2}{*}{\multicolumn{3}{|c|}{ Non-intervention group }} \\
\hline & \multirow[b]{2}{*}{$\begin{array}{l}\text { Baseline } \\
\text { mean }\end{array}$} & \multicolumn{2}{|c|}{$\begin{array}{l}\text { Subgroup A with one } \\
\text { intervention }\end{array}$} & \multicolumn{2}{|c|}{$\begin{array}{l}\text { Subgroup B with two } \\
\text { interventions }\end{array}$} & & & \\
\hline & & $\begin{array}{l}12 \text { months } \\
\text { mean }\end{array}$ & $\begin{array}{l}\text { Group } \\
\text { change } \\
\text { effect size }\end{array}$ & $\begin{array}{l}12 \text { months } \\
\text { mean }\end{array}$ & $\begin{array}{l}\text { Group } \\
\text { change } \\
\text { effect size }\end{array}$ & $\begin{array}{l}\text { Baseline } \\
\text { mean }\end{array}$ & $\begin{array}{l}12 \text { months } \\
\text { mean }\end{array}$ & $\begin{array}{l}\text { Group } \\
\text { change } \\
\text { effect size }\end{array}$ \\
\hline $\begin{array}{l}\text { Health directed } \\
\text { behaviour }\end{array}$ & 2.82 & 3.09 & $0.50^{* *}$ & 3.35 & $0.99^{* * *}$ & 2.73 & 2.65 & -0.22 \\
\hline $\begin{array}{l}\text { Positive and active } \\
\text { engagement in life }\end{array}$ & 2.84 & 3.09 & $0.54^{* *}$ & 3.20 & $0.81^{* * *}$ & 2.80 & 2.83 & 0.07 \\
\hline $\begin{array}{l}\text { Self-monitoring and } \\
\text { insight }\end{array}$ & 2.97 & 3.14 & $0.36^{*}$ & 3.21 & $0.48^{*}$ & 2.96 & 2.89 & -0.17 \\
\hline $\begin{array}{l}\text { Constructive } \\
\text { attitudes \& } \\
\text { approaches }\end{array}$ & 2.92 & 3.16 & $0.40^{*}$ & 3.23 & $0.50^{* *}$ & 2.96 & 2.81 & -0.33 \\
\hline $\begin{array}{l}\text { Skills and technique } \\
\text { acquisition }\end{array}$ & 2.78 & 3.10 & $0.59^{* *}$ & 3.23 & $0.73^{* *}$ & 2.75 & 2.95 & $0.43^{*}$ \\
\hline $\begin{array}{l}\text { Social integration \& } \\
\text { support }\end{array}$ & 2.86 & 3.11 & $0.44^{*}$ & 3.17 & $0.54^{* *}$ & 2.84 & 2.86 & 0.05 \\
\hline $\begin{array}{l}\text { Health services } \\
\text { navigation }\end{array}$ & 2.66 & 3.15 & $0.85^{\star \star *}$ & 3.14 & $0.88^{* * * x}$ & 2.64 & 2.85 & $0.55^{* *}$ \\
\hline Emotional wellbeing & 2.40 & 2.13 & $-0.45^{*}$ & 2.01 & $-0.68^{* *}$ & 2.41 & 2.45 & 0.06 \\
\hline
\end{tabular}

"small effect size; ${ }^{* *}$ medium effect size; ${ }^{* * *}$ greater effect size. 
months except for skills and technique acquisition between baseline to six months among intervention group in comparison to the non-intervention group (control group) (Table 2).

Further analysis results of subgroups $A$ and $B$ of intervention group and non-intervention group summarised in Table 3 indicate the net group change effect size of the knowledge improvement calculated based on questionnaire developers' guidelines.

The effect size in each domain after the repeated education intervention (at 12 months) is summarised in Table 3. Domain 2: Positive and active engagement in life and Domain 1: Health directed behaviour showed greater improvement following the second follow up than the other domains. Domain 5: Constructive attitudes and approaches, Domain 7: Social integration and support and Domain 3: Emotional wellbeing showed improvement in the small to medium range. Although a positive effect was evident in the answers for Domain 4: Self-monitoring and insight, it was small.

As summarised in Table 3, Domain 8: Health services navigation and Domain 6: Skills and technique acquisition showed small and medium effect size changes while majority of the domains with quite low or negative effect.

Analysis of the second follow up, after 12 months, revealed a reduction of net positive reliable change in domains in the non-intervention group.

The assessment of health education program based on nine follow-up questions from those who completed the education interventions, mean score was calcula- ted. Figure 2 shows that Question 45 "It was worth my time to take part in this study" scored the highest, with a score of 5.55, while all the questions received an average score of more than five. This finding confirms the success of the education intervention.

\section{Discussion}

The findings from the baseline analysis indicated that all participants, intervention and non-intervention, shared a similar starting point with their health and physical measurements and diabetes knowledge.

The results of this study reveal that all heiQ ${ }^{\mathrm{TM}}$ domains showed at least low to moderate correlations with the follow ups. The initial mean values in the current study were comparative to the results presented in the French translated hei $\mathrm{Q}^{\mathrm{TM}}$ data $[9,18]$. This difference may indicate that the Sri Lankan participants already had limited knowledge of their disease. However, this exploration of the positive improvement in all domains in the intervention group during the follow-up analysis compared to the non-intervention group, strongly evidences the effect of the education intervention on knowledge improvement in the study participants. Domains 1, 3 and 6 showed higher results in this study than that of the previous studies validated in the French translated heiQ ${ }^{\mathrm{TM}}$. However, results were in a middle range between the two studies for Domains 2, 4, 5, 7 and 8.

A study by Osborne, et al., the developer of the hei$\mathrm{Q}^{\mathrm{TM}}$, concluded that it is common for people who accumulate a high heiQ ${ }^{\mathrm{TM}}$ score in Domain 3: Emotional wel-

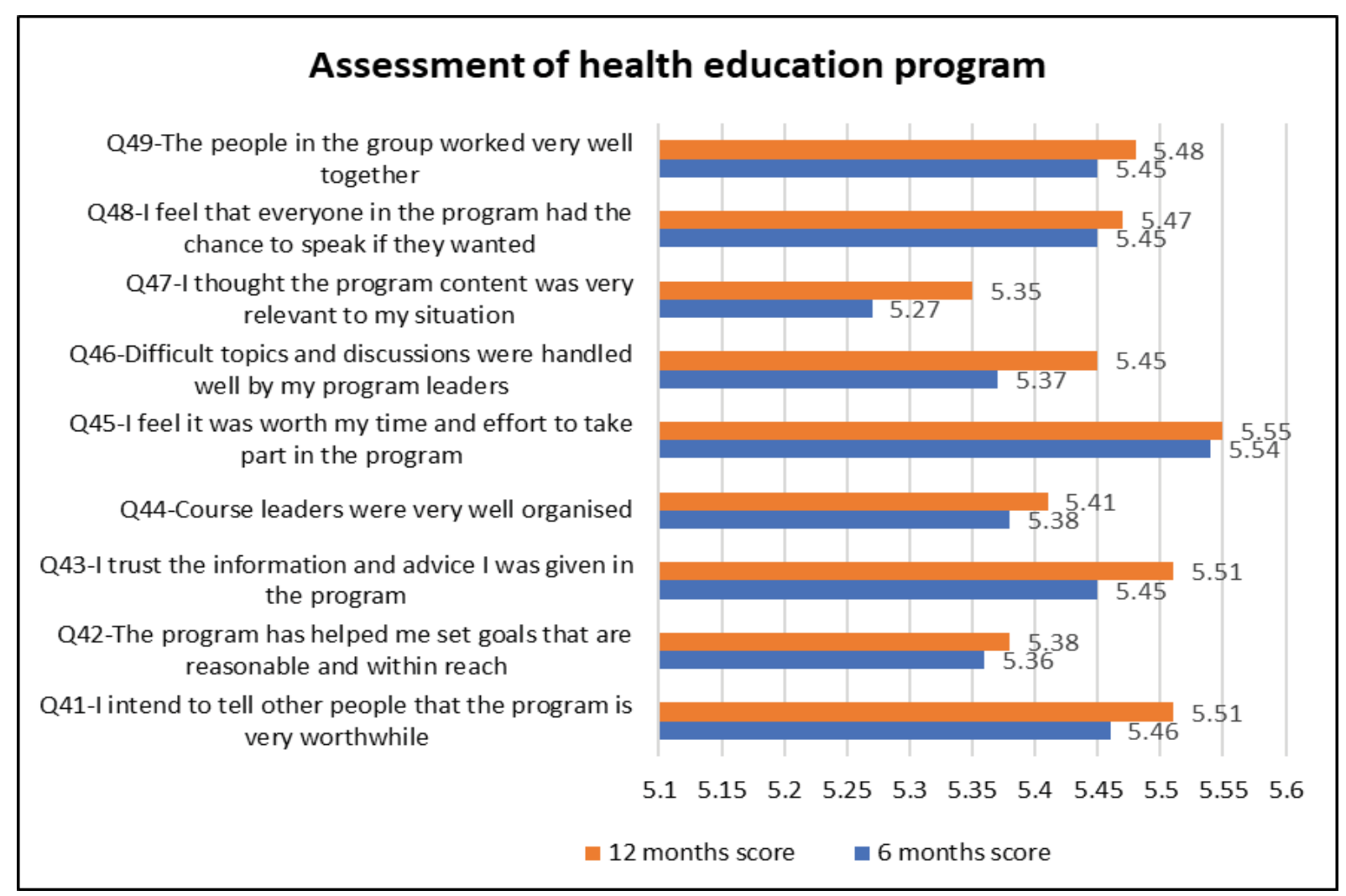

Figure 2: Evaluation of the health-education program. 
Ibeing to also have high scores in Domain 2: Positive and active engagement in life and a low score in Domain 4: Self-monitoring and insight. This current study validated this finding. The study sample revealed that Domains 8 and 7: Health services navigation and Social integration and support respectively, had the lowest scores. This result might be due to the poor development of technology, or less priority given to personal conditions than family matters as per cultural beliefs in adults in Sri Lanka. These findings evidence by the Amarasekara, et al. 2014 study of health beliefs and practices in Sri Lanka.

The greatest effect size was found in Domain 1: Health directed behaviour, Domain 2: Positive and active engagement in life and Domain 8: Health services navigation, between the baseline score and the follow ups in the intervention group. Domain 5: Constructive attitudes and approaches, Domain 6: Skills and technique acquisition, Domain 7: Social integration and support and Domain 3: Emotional wellbeing were also notable in the way intervention group individuals participated in the education session in the follow ups compared to that delivered at baseline. Intervention group participants also showed an improvement in their ability to confidently interact with a range of health organisations and health professionals (the researcher, nurses, GPs and the pathology laboratory). These findings are critical for patients in Sri Lanka, as they have high dependence on physicians' instructions and on medical healthcare professionals to manage their health conditions. This could increase the overcrowded issues in most state sector hospitals in Sri Lanka as revealed by the Medagama, et al., 2015 study. In addition, these findings reveal that the participants were encouraged to become proactive in managing their health.

However, there was a slight reduction in skill and technique acquisition and health service navigation at six months, after twelve months. These findings seem reasonable as people with worse health may have had more experience or challenge with these areas and been more engaged with health professionals to control their conditions, whilst they become less responsive when they get used to their disease condition.

There was no revision made to the translated version of the heiQ ${ }^{\mathrm{TM}}$ as all items precisely promote health and behavioural change. However, based on verbal communication with the study participants, the terminology used the items translated from "I feel hopeless because of my health problems" and "I feel like I am actively involved in my life" were shown not to be fully understood, and they were answered after clarification by the investigator. Some clarification was made to reduce variability in results. Therefore, further research is encouraged using the modifications to the translation of those two statements.

The follow-up questions were based on the fundamental environmental and personal determinants of a person's opportunities to access the research intervention and provide their feedback. Question 45: "It was worth my time to take part in this study" scored the highest, with a score of 5.58. It was one of the main outcomes of the education intervention that had positive feedback and highlights the success of the education intervention.

\section{Conclusion}

The heiQ ${ }^{\mathrm{TM}}$ translated into Sinhala was well accepted by participants suffering from T2DM, revealing the usefulness of the questionnaire as an instrument to evaluate the impact of health-education intervention for any chronic disease, not just diabetes. Sinhala heiQ ${ }^{\mathrm{TM}}$ serves as a proximal goal for self-management programs to advance outcome assessment in this field in Sri Lanka.

\section{Practical Implications and Recommendations}

The method used in this study through the use of the heiQ ${ }^{\text {TM }}$ can identify individual patient education needs and address relevant aspects to their education status and health education gaps. Thus, current study intervention could be used as a model of an integrated program to improve T2DM patient awareness and medication self-management by incorporating it to the current T2DM management program. Additionally, the heiQ ${ }^{\mathrm{TM}}$ could be used as a tool for further studies to determine the value of its application in other health conditions, to compare outcomes across multiple cultures and languages in Sri Lanka.

\section{Acknowledgements}

I would like to thank all medical, nursing and laboratory staff; and all participants from Karapitiya Teaching Hospital and Colombo South Teaching Hospital, Kalubowila. Special thanks to Charles Darwin University, Australia and University Grant Commission Sri Lanka for providing scholarship and grants to support for first author's study.

\section{Declaration of Conflicting Interests}

The authors declare no known conflict of interest related to this article.

\section{Registration of Study}

This study was registered under the Sri Lanka Clinical Trial Registry, No: SLCTR/2015/014, which is a primary registry for clinical trials involving human subjects conducted in Sri Lanka that is linked to the Registry Network of the International Clinical Trials Registry Platform of the WHO. The site approval was obtained from Directors of both tertiary care facilities in Sri Lanka.

\section{Ethical Approval}

The study was approved by the Human ethics committee (Ref. No: HI4082) of Charles Darwin University, Australia and Ethics Review Committee (Ref No: 17.11.2014:3.32) of Faculty of Medicine, University of Ruhuna, Sri Lanka. 


\section{References}

1. Katulanda P, Constantine GR, Mahesh JG, Sheriff R, Seneviratne RD, et al. (2008) Prevalence and projections of diabetes and pre-diabetes in adults in Sri Lanka--Sri Lanka Diabetes, Cardiovascular Study (SLDCS). Diabet Med 25: 1062-1069.

2. Amarasekara AA, Fongkaew W, Turale S, Wimalasekara SW, Chanprasit C (2014) An ethnographic study of diabetes health beliefs and practices in Sri Lankan adults. Int Nurs Rev 61: 507-514.

3. International Diabetes Federation (2017) IDF Diabetes Atlas. ( $8^{\text {th }}$ edn), Brussels, Belgium International Diabetes Federation.

4. Jayasuriya R, Pinidiyapathirage MJ, Jayawardena R, Kasturiratne A, De Zoysa P, et al. (2015) Translational research for Diabetes Self-Management in Sri Lanka: A randomized controlled trial. Prim Care Diabetes 9: 338-345.

5. Osborne RH, Batterham R, Livingston J (2011) The evaluation of chronic disease self-management support across settings: the international experience of the health education impact questionnaire quality monitoring system. Nurs Clin North Am 46: 255-270.

6. Choi TST, Walker KZ, Ralsto RA, Claire Palermo (2015) Diabetes education needs of Chinese Australians: A qualitative study. Health Education Journal 74: 197-208.

7. Jordan JE, Osborne RH, Buchbinder R (2011) Critical appraisal of health literacy indices revealed variable underlying constructs, narrow content and psychometric weaknesses. J Clin Epidemiol 64: 366-379.

8. Osborne RH, Elsworth GR, Whitfield K (2007) The Health Education Impact Questionnaire (heiQ): An outcomes and evaluation measure for patient education and self-management interventions for people with chronic conditions. Patient Educ Couns 66: 192-201.

9. Morita R, Arakida M, Osborne RH, Nolte S, Elsworth GR, et al. (2013) Adaptation and validation of the Japanese version of the Health Education Impact Questionnaire (heiQ-J) for the evaluation of self-management education interventions. Jpn J Nurs Sci 10: 255-266.
10. Epstein J, Osborne RH, Elsworth GR, Beaton DE, Guillemin $F(2015)$ Cross-cultural adaptation of the Health Education Impact Questionnaire: Experimental study showed expert committee, not back-translation, added value. J Clin Epidemiol 68: 360-369.

11. Osborne RH, Batterham RW, Elsworth GR, Hawkins M, Buchbinder $R$ (2013) The grounded psychometric development and initial validation of the Health Literacy Questionnaire (HLQ). BMC Public Health 13: 658-675.

12. Elsworth GR, Nolte S, Osborne RH (2015) Factor structure and measurement invariance of the Health Education Impact Questionnaire: Does the subjectivity of the response perspective threaten the contextual validity of inferences? SAGE Open Med 3: 1-13.

13. Pinidiyapathirage MJ, Kasturiratne A, Ranawaka UK, Gunasekara D, Wijekoon N, et al. (2013) The burden of diabetes mellitus and impaired fasting glucose in an urban population of Sri Lanka. Diabet Med 30: 326-332.

14. Ranasinghe $D C$, Ranasinghe $P$, Jayawardena $R$, Matthews DR, Katulanda P (2014) Evaluation of physical activity among adults with diabetes mellitus from Sri Lanka. Int Arch Med 7: 15-19.

15. Medagama AB, Bandara R, Wijethunga R (2015) The high burden of in-hospital diabetes mellitus at a tertiary care hospital in Sri Lanka: A case control study. J Diabetes Metab 6: 502-506.

16. Cooray BPR, Ball PA, Morrissey H, Waidyarathne El (2017) The effect of health education in the management of type 2 Diabetes and the Sri Lankan perspective: A Review. Sri Lanka Journal of Diabetes Endocrinology and Metabolism 7: 33-38.

17. Stewart NM (2012) Practical Patient Literacy: The Medagogy Model. ( $1^{\text {st }}$ edn), McGraw-Hill Education/Medical.

18. Belanger A, Hudon C, Fortin M, Amirall J, Bouhali T, et al. (2015) Validation of a French-language version of the health education impact Questionnaire (heiQ) among chronic disease patients seen in primary care: A cross-sectional study. Health and Quality of Life Outcomes 13: 64. 